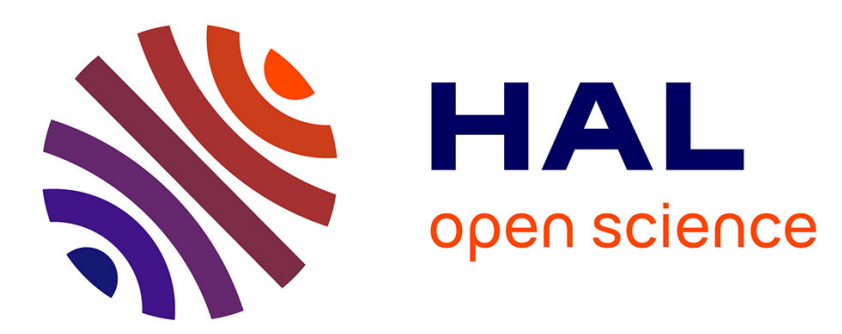

\title{
Application of hybrid linear ion trap-high resolution mass spectrometry to the analysis of mycotoxins in beer
} Josep Rubert, Kevin J. James, Carla Soler

\section{To cite this version:}

Josep Rubert, Kevin J. James, Carla Soler. Application of hybrid linear ion trap-high resolution mass spectrometry to the analysis of mycotoxins in beer. Food Additives and Contaminants, 2011, pp.1. 10.1080/19440049.2011.595015 . hal-00719819

\section{HAL Id: hal-00719819 \\ https://hal.science/hal-00719819}

Submitted on 21 Jul 2012

HAL is a multi-disciplinary open access archive for the deposit and dissemination of scientific research documents, whether they are published or not. The documents may come from teaching and research institutions in France or abroad, or from public or private research centers.
L'archive ouverte pluridisciplinaire HAL, est destinée au dépôt et à la diffusion de documents scientifiques de niveau recherche, publiés ou non, émanant des établissements d'enseignement et de recherche français ou étrangers, des laboratoires publics ou privés. 


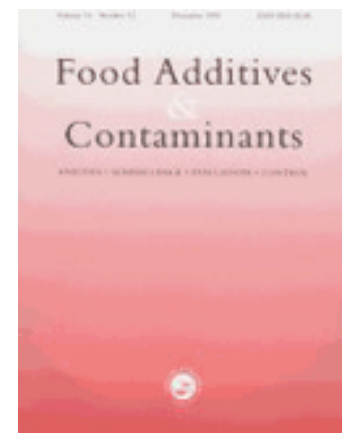

\section{Application of hybrid linear ion trap-high resolution mass spectrometry to the analysis of mycotoxins in beer}

\begin{tabular}{|r|l|}
\hline Journal: & Food Additives and Contaminants \\
\hline Manuscript ID: & TFAC-2011-092.R1 \\
\hline Manuscript Type: & Original Research Paper \\
\hline Author: & 03-Jun-2011 \\
\hline Complete List of Authors: & $\begin{array}{l}\text { Rubert, Josep } \\
\text { James, Kevin } \\
\text { Soler, Carla; Universitat de Valencia, Medicina Preventiva }\end{array}$ \\
\hline Additives/Contaminants: & Lycotoxins \\
\hline Food Types: & Beer \\
\hline & $\begin{array}{l}\text { This paper presents the application of liquid chromatography } \\
\text { electrospray ionization ion trap - orbitrap mass spectrometry for the } \\
\text { determination of } 18 \text { mycotoxins (aflatoxins, fumonisins, } \\
\text { trichothecens, ochratoxin A, sterogmatocistine, beauvaricin, } \\
\text { zearalenone and zearalenol) in beer. The extraction procedure was } \\
\text { carried out by solid phase extraction (SPE): SPE columns were } \\
\text { conditioned with acetonitrile/methanol and water. Then, beer was } \\
\text { loaded and the SPE columns were washed with water. In these } \\
\text { conditions, the recoveries were higher than 65 \% and the relative } \\
\text { standard deviation (RSD) were lower than 18 \%. The lowest limits } \\
\text { of quantification (LLOQ) ranged from 9 to 155 ng ml-1. Matrix- } \\
\text { matched calibration was performed for each beer, and reliable } \\
\text { results were obtained from selected mycotoxins. The method was } \\
\text { applied to the analysis of 25 commercial beers. Taking advantage of } \\
\text { the hybrid capabilities, the presence of other mycotoxins were } \\
\text { checked; enniatins (A, A1, B and B1) and fusaproliferin were } \\
\text { studied in all the tested samples. } \\
\text { The results confirmed the presence of zearalenone in one stout beer } \\
\text { sample. }\end{array}$ \\
\hline Abstract \\
\hline
\end{tabular}


1

2

4

5

6

7

8

9

10

11

12

13

14

15

16

17

18

19

20

21

22

23

24

25

26

27

28

29

30

31

32

33

34

35

36

37

38

39

40

41

42

43

44

45

46

47

48

49

50

51

52

53

54

55

56

57

58

59

60

\section{SCHOLARONE \\ Manuscripts}

http://mc.manuscriptcentral.com/tfac Email: fac@tandf.co.uk 


\section{Application of hybrid linear ion trap-high resolution mass}

2 spectrometry to the analysis of mycotoxins in beer

3

$4 \quad$ J. Rubert $^{\mathrm{a}}$, J. Mañes ${ }^{\mathrm{a}}$, K.J. James ${ }^{\mathrm{b}}$, C. Soler ${ }^{\mathrm{a} *}$

5

6

7 a Department de Medicina Preventiva I Salut Pública. Facultat de Farmàcia. Universitat

8 de Valéncia. Av. Vicent Andrés Estellés 46100 Burjassot (Spain);

$9{ }^{b}$ PROTEOBIO. Mass spectrometry laboratory. Cork Institute of Technology. Rose

10 Avenue, Cork (Ireland).

11

12

13

14

15

16

17

18

19

20

21

$22 *$ Correspondence to: C. Soler, Department of Preventive Medicine and Public Health.

23 Facultat de Farmàcia. Universitat de València. Av. Vicent Andrés Estellés 46100

24 Burjassot (Spain).

25 E-mail: carla.soler@uv.es 
26 Abstract

27 This paper reports the application of liquid chromatography electrospray ionization ion

28 trap - orbitrap mass spectrometry for the determination of 18 mycotoxins (aflatoxins,

29 fumonisins, trichothecenes, ochratoxin A, sterogmatocystin, beauvaricin, zearalenone

30 and zearalenol) in beer. The extraction procedure was carried out by solid phase

31 extraction (SPE): SPE columns were conditioned with acetonitrile/methanol and water.

32 Beer was loaded onto the column which was washed with water. In these conditions, the

33 recoveries were more than $65 \%$ and the relative standard deviation (RSD) were below

$3418 \%$. The lowest limits of quantification (LLOQ) ranged from 9 to $155 \mathrm{ng} \mathrm{ml}^{-1}$. Matrix-

35 matched calibration was performed for each beer, and reliable results were obtained

36 from selected mycotoxins. The method was applied to the analysis of 25 commercial

37 beers. Taking advantage of the hybrid capabilities, the presence of other mycotoxins were checked; enniatins $\left(A, A_{1}, B\right.$ and $\left.B_{1}\right)$ and fusaproliferin were studied in all the tested samples. The survey detected the presence of zearalenone in one stout beer sample.

50 Keywords: mycotoxins; Orbitrap; beer; high resolution mass; SPE 


\section{Introduction}

52 During the 1970s, considerable efforts were directed toward perfecting high resolution

53 instruments. Fortunately, nowadays when liquid chromatography (LC) is combined with

54 a high resolution instrument, it can support a wide range of applications from routine

55 compound identification in complex mixtures (Thurman et al. 2006). One of these high

56 resolution instruments is LTQ Orbitrap MS; it combines a high resolution mass

57 spectrometer, as Orbitrap analyzer, with an external accumulation device such as a

58 linear ion trap, making possible multiple levels of fragmentation $\left(\mathrm{MS}^{\mathrm{n}}\right)$ for the

59 elucidation of analyte structures. The use of the LTQ Orbitrap allows high-quality

60 accurate mass and acquisition of $\mathrm{MS}^{\mathrm{n}}$ spectra. Fourier transformation of the acquired

61 transient allows wide mass range detection with high resolving power, mass accuracy,

62 and dynamic range. The LTQ Orbitrap MS has two main advantages: high sensitivity in

63 full scan and possibility of determination of accurate mass of product ions (Makarov et

64 al. 2010).

65

66 In the last years, the use of this instrument has been restricted to proteomic or metabolic

67 field (Bennett et al. 2011). In fact, there is scarce literature about its application in the

68 analysis of molecules with relative low molecular weight such as pesticides (Edison et

69 al. 2011), veterinary drugs and doping control (van der Heeft et al. 2009; Peters et al.

70 2010) or toxins. Hogenboom et al. (2009) applied Orbitrap technology to search for

71 target compounds and "unknown" compounds in different water samples. This study

72 demonstrated the presence of target compounds as pharmaceuticals, benzotriazoles and

73 illicit drugs, and they identified "unknown" compounds in groundwater sample and

74 landfill soil sample. Škrabáková et al. (2010) validated the use of high mass resolution

75 in full-scan mode for the determination of six azaspiracids in mussel tissue extracts. 
76 In the field of mycotoxins analysis, Herebian et al. (2009) carried out a first approach

77 for determining 31 mycotoxins in grain and they compared QqQ technology with

78 Orbitrap instrument. They concluded that one of the major advantages of the high

79 resolution full scan method is the possibility of screening unknown compounds.

80 However, best sensitivity was obtained with triple quadrupole (QqQ).

81

82 Improving the results, Vaclavik et al. (2010) used Orbitrap technology to determine 11

83 mycotoxins in cereals and the same group extended the analysis to 32 mycotoxins in

84 beer (Zachariasova et al. 2010). In the first study QuEChERS was used for extraction,

85 while in the second study the extraction was carried up by classical liquid-liquid 86 extraction.

87

88 Therefore, expanding upon this previous work, the objectives of the present study were;

89 (i) to develop and to validate an analytical procedure based on the applicability of the

90 LTQ Orbitrap for the simultaneous extraction of aflatoxins, fumonisins, trichothecenes,

91 OTA, ZEN, ZON and BEA in beer. (ii) to apply the validated method for the routine

92 analysis of 25 different Irish beer samples collected from Cork (Ireland). (iii) To apply

93 the method to acquire data essential for the identification of other "non-target"

94 mycotoxins that may be present in beer:. enneatins $\left(A, A_{1}, B\right.$ and $\left.B_{1}\right)$ and fusaproliferin

95 were checked.

97 Materials and methods

$98 \quad$ Reagents and chemicals

99 HPLC grade solvents, acetonitrile, methanol and water were supplied by ThermoFisher

100 (Dublin, Ireland). Analytical grade reagent formic acid (purity > 98\%) ammonium 
101 formate was obtained from Panreac Quimica S.A.U. (Barcelona, Spain). All solvents

102 were passed through a $0.45 \mu \mathrm{m}$ cellulose filter purchased from Scharlau (Barcelona,

103 Spain).

104 Stock solution

105 The certificate standards of aflatoxin $B_{1}\left(A_{F B}\right)$, aflatoxin $B_{2}\left(A_{F B}\right)$, aflatoxin $G_{1}$

$106\left(\mathrm{AFG}_{1}\right)$, aflatoxin $\mathrm{G}_{2}\left(\mathrm{AFG}_{2}\right)$, Ochratoxin $\mathrm{A}(\mathrm{OTA})$, sterigmatocystin (STER), $\alpha-$

107 zearalenol (ZOL), zearalenone (ZEN), nivalenol (NIV), deoxynivalenol (DON), 3-

108 acetyldeoxynivalenol (3-ADON), 3-acetyldeoxynivalenol-3deuterated (3-ADON-d 3 ,

109 diacetoxyscirpenol (DAS), neosolaniol (NEO), fumonisin $\mathrm{B}_{1}\left(\mathrm{FB}_{1}\right)$, fumonisin $\mathrm{B}_{2}\left(\mathrm{FB}_{2}\right)$

110 and beauvericin (BEA) were purchased from Sigma Aldrich (Madrid, Spain). T-2 and

111 HT-2 toxin and aflatoxin $\mathrm{M}_{1}\left(\mathrm{AFM}_{1}\right)$ stock solutions (in acetonitrile) were obtained

112 from Biopure referenzsubstanzen $\mathrm{GmBH}$ (Tulln, Austria). Fumonisin $\mathrm{B}_{3}\left(\mathrm{FB}_{3}\right)$ was

113 supplied by the PROMEC unit (Programme on Mycotoxins and Experimental

114 Carcinogenesis, Tygerberg, South Africa).

115

116 The individual stock solutions of aflatoxins (AFs) and OTA with concentration 500

$117 \mu \mathrm{g} / \mathrm{ml}$ were prepared in acetonitrile and STER, ZOL, ZEN, NIV, DON, 3-ADON, FB ${ }_{1}$,

$118 \mathrm{FB}_{2}$, BEA were prepared on the same concentration in methanol. On the one hand, stock

119 solutions $\mathrm{FB}_{3}$, DAS, T-2 and HT-2 at concentration of $100 \mu \mathrm{g} / \mathrm{ml}$ were prepared in

120 acetonitrile. As internal standard (IS) compounds $\mathrm{AFM}_{1}$ at $0.05 \mu \mathrm{g} \mathrm{ml}^{-1}$ and 3-ADON-

$121 \mathrm{~d}_{3}, \mathrm{NEO}$ at $0.150 \mu \mathrm{g} \mathrm{ml}^{-1}$ were used; working solutions were prepared by dilution of

122 individual stock solutions in methanol. All the standard solutions were kept in safety

123 conditions and at $-20^{\circ} \mathrm{C}$. 
125 All other working standard solutions were prepared immediately before use by diluting

126 the stock solution with methanol:water (50:50) (V/V).

127

128 Oasis HLB SPE (200 mg) cartridges were purchased from Waters (Milford, MA, USA).

129 An extraction manifold from Waters connected to a Büchi Vac V-500 (Flawil,

130 Switzerland) vacuum system was used for SPE experiments.

132 Samples

133 Beer samples were purchased from different stores and pubs of Cork (Ireland) and kept

134 at $-20^{\circ} \mathrm{C}$ in a dark and dry place. The bottle samples were purchased in supermarkets

135 and conserved. On the other hand, pint $(568 \mathrm{ml})$ samples were collected in sterile

136 beakers from each individual beer and stored until analysis. Samples with undetectable

137 levels of mycotoxins were used for spiking and recovery studies.

138

\section{Extraction Procedure}

140 The extraction procedure was developed according to Romero-Gonzalez et al. (2009).

141 Beer was degassed by sonication for $25 \mathrm{~min}$. The Oasis HLB cartridges were

142 conditioned with $5 \mathrm{ml}$ of acetonitrile/methanol $(50: 50 \mathrm{v} / \mathrm{v})$ and $5 \mathrm{ml}$ of water.

143 Consequently, $10 \mathrm{ml}$ of beer was loaded onto $\mathrm{C}_{18}$ cartridge. After that, SPE columns

144 were washed with $5 \mathrm{ml}$ of water. Then the cartridges were dried for $30 \mathrm{~min}$. In the last

145 step, the mycotoxins were eluted by adding $4 \mathrm{ml}$ of acetonitrile/methanol $(50: 50 \mathrm{v} / \mathrm{v})$.

146 Then, the extract was transferred into a $15 \mathrm{ml}$ conical tube and evaporated to dryness at

$14735{ }^{\circ} \mathrm{C}$ with a gentle stream of nitrogen using a multi-sample Turbovap LV Evaporator

148 (Zymark, Hoptkinton, USA). The residue was reconstituted to a final volume of $1 \mathrm{ml}$ 
149 with methanol/water (50:50) (v/v), filtered through a $0.20 \mu \mathrm{m}$ Millex-GN nylon filter

150 (Millipore, Carrightwohill, Ireland) and collected into a vial.

151

152 HPLC-Orbitrap XL

153 Chromatographic separations were carried out using an Accela LC system (Thermo

154 Scientific, Hemel Hempstead, UK), equipped with a reversed-phase analytical column

155 (Gemini $\mathrm{C}_{18},, 150 \mathrm{~mm}, 2 \mathrm{~mm}$ i.d, $5 \mu \mathrm{m}$; Phenomenex) maintained at $35^{\circ} \mathrm{C}$. As mobile 156 phase, $5 \mathrm{mM}$ ammonium formate and $0.1 \%$ formic acid in water (A) and $5 \mathrm{mM}$

157 ammonium formate in methanol (B) were used. The gradient was as follows: at the start

$1585 \%$ solvent B was used: the percentage of solvent B was linearly increased up to $95 \%$

159 in $10 \mathrm{~min}$ and then the percentage of solvent B was linearly decreased to $80 \%$ in 5 min.

160 The column was equilibrated to initial conditions for $5 \mathrm{~min}$. The flow rate was $200 \mu \mathrm{l}$

$161 \min ^{-1}$ and the injection volume was $10 \mu \mathrm{l}$.

162

163 The LC system was connected to a hybrid LIT-FT mass spectrometer (LTQ Orbitrap

164 XL; Thermo Scientific, Bremen, Germany), operating in positive ion mode. The linear

165 ion trap (LTQ) part of the hybrid MS system was equipped with heated electrospray

166 interface (H-ESI). Full-scan accurate mass spectra (mass range from 90 to 900 Da) were

167 obtained at high resolution (100,000 FWHM) and processed using Xcalibur v.2.0

168 software. The electrospray source conditions were: source voltage $4 \mathrm{kV}$, heated

169 capillary temperature $275^{\circ} \mathrm{C}$, capillary voltage $30 \mathrm{~V}$, sheat gas and auxiliar gas 35 and

17030 (arbitrary units), respectively.

171

172 The mass spectrometer was operated in a data-dependent-acquisition (DDA) mode in

173 which both $\mathrm{MS}$ and $\mathrm{MS}^{\mathrm{n}}$ spectra were acquired specifying parent masses of target 
174 compounds. In this mode, the acquisition software probed the MS spectra in real-time 175 on a full scan allowed accurate mass. The accurate mass is capable to find true 176 unknowns since the method does not require any pre-selection of masses. The

177 instrument is initially set to operate in full-scan mode until a parent ion appears to preset 178 the instrument, which switches into the product-ion mode $\left(\mathrm{MS}^{\mathrm{n}}\right)$. The mass resolution 179 was set at 100,000 FWHM for both screening and quantitative analysis. The products 180 ions were generated in the LTQ trap at an optimized collision energy setting of 181 mycotoxins. The scan type settings are presented in Table 1. No exclusion list was used.

182 The total cycle time depends upon the resolution; at a resolution of 100,000 FWHM the 183 total cycle time is about $1 \mathrm{~s}$. The results were used to create a (full-scan) accurate mass 184 MS and an $\mathrm{MS}^{\mathrm{n}}$ database to enable identification of compound in future screening.

185 The Orbitrap instrument was calibrated using a solution containing caffeine, MRFA, 186 and Ultramark 1621, according to the manufacturer's instructions. Operation of the 187 entire LC/MS instrumentation was controlled using Xcalibur software (Thermo 188 Scientific).

190 Data processing

191 LTQ Orbitrap MS for target compound analysis is related to its high mass resolution 192 potential, corresponding to a mass assignment accurate to four decimal places. The 193 ability to determine the $\mathrm{m} / \mathrm{z}$ of an ion to within a maximum deviation of $5 \mathrm{ppm}$ allows 194 the determination of a unique elemental composition based on the mass defect of the 195 constituent atoms (van der Heeft et al. 2009). The ability to closely match the 196 measured/theoretical mass with the observed mass greatly increases the reliability of 197 identification (table 1). 
199 The total list of accurate masses detected in a sample was corrected manually for masses

200 also found in matrix. Based on the accurate mass, the elemental composition of the 201 peaks of interest was calculated using the elemental composition tool within the 202 Xcalibur software.

203

204 In this study, the calculated elemental compositions with a maximum deviation of 5

205 ppm from the measured exact mass were considered. To ensure correct mass calibration, 206 the exact mass of the MS internal standard compounds were checked regularly. To

207 obtain an elemental composition, all compounds were studied by chemical structure. So, 208 theoretical mass was calculated by using ChemDraw 11.0 (CambridgeSoft) and 209 www.chemspider.com.

210

211 Accurate mass screening for non target mycotoxins

212 Full-scan accurate mass measurements were compared with theoretical exact masses of

213 known emergent mycotoxins, such as enniatins $\mathrm{A}, \mathrm{B}, \mathrm{A}_{1}, \mathrm{~B}_{1}$ and fusaproliferin (Jestoi et 214 al. 2004; Jestoi 2008; Jestoi et al 2009).

215

216 Method Validation

217 Confirmation of the identity was carried out by comparing the retention time and 218 fragmentation pattern of the compound with that of a certified standard and 219 quantification by accurate mass (European Community 2002). Linearity was assessed 220 using standard solutions and matrix-matched calibrations by analyzing in triplicate six 221 concentrations levels; matrix-matched calibration was prepared for each beer brand. To 222 evaluate matrix effects, two strategies were studied: matrix-matched calibration and 223 internal standard addition. The recovery experiments were carried out by spiking the 
224 sample in five replicates at two concentration levels: LLOQ level and 100 times LLOQ

225 level (Table 2). In the same way, precision of the method was determined in fortified 226 beer at LLOQ level and 100 times LLOQ level and calculated as RSD of measurements 227 in quintuplicate, in the same day and five non-consecutive days.

229 Other analytical parameters, such as limits of detection (LODs) and lowest limits of 230 quantification (LLOQs), were calculated by Xcalibur 2.0 software; both parameters 231 were determined by decreasing concentration levels in fortified beer. When the signal232 to-noise ratios $(\mathrm{S} / \mathrm{N})$ of 3 or above were considered acceptable for LOD. LLOQ was 233 defined as the lowest concentration that could be quantified with coefficient of variation $234(\% \mathrm{CV})$ and the absolute value of mean relative error (\%MRE) [(spiked 235 concentration-nominal concentration/nominal concentration) $\times 100$ ] of less than or 236 equal to $20 \%$. (table 2 ).

237

238 RESULTS AND DISCUSSION

239 LC-LTQ Orbitrap optimization.

240 All ion source tune parameters were optimized manually by infusing mycotoxin 241 standards and monitoring the $\left[\mathrm{M}+\mathrm{NH}_{4}\right]^{+}$ion for trichothecenes type $\mathrm{A}$ and BEA, also $242[\mathrm{M}+\mathrm{H}]^{+}$ion for the other mycotoxins (table 1). This optimization was carried out by 243 adjusting the parameters until no precursor ion fragmentation was observed. In this 244 study mass resolution was set at 100000 FWHM and the mean values of the mass 245 measurements were all lower than $3 \mathrm{ppm}$ of their theoretical $\mathrm{m} / \mathrm{z}$ values, as it is shown 246 in table 1. 
248 Table 1 presents also the data-depending acquisition settings. $\mathrm{MS}^{\mathrm{n}}$ measurements were

249 performed to obtain information of main fragment ions generated in the linear ion trap

250 (nominal mass product ions) within the same analysis. In addition, the accurate masses

251 of these product ions were linked with precursor compound masses.

252 Confirmation of the identity was done by comparing the retention time, accurate mass

253 and $\mathrm{MS}^{\mathrm{n}}$ fragmentation pattern of the compound to that of a reference standard.

SPE Optimization.

256 The extraction was carried out according to Romero-Gonzalez et al. (2009). However, 257 in this study the range of mycotoxin analyzed was extended and, in order to improve the 258 recovery values, some parameters were studied. With this aim, blank beer was fortified 259 at 2 times LLOQ level being this level the reference concentration level.

261 The organic extraction solvent was the parameter optimized due to the wide-range of 262 mycotoxins analyzed with different polarities. Then, the use of $4 \mathrm{~mL}$ of methanol and 4 $263 \mathrm{ml}$ of acetonitrile were compared. Methanol provided good recoveries for fumonisins $264\left(\mathrm{FB}_{1}, \mathrm{FB}_{2}\right.$, and $\left.\mathrm{FB} 3\right)$ and trichothecenes type B (DON, NIV, 3-ADON); however, the 265 recoveries obtained for trichothecenes type A and AFs were lower than $50 \%$. On the 266 other hand, acetonitrile reached acceptable recovery values for AFs and trichothecenes 267 Type A (>65\%). For this reason, it was decided to use a solvent mixture elution of 268 acetonitrile/methanol. The proportion acetonitrile/methanol (40:60 v/v) proposed by 269 Romero et al. (2009) allowed acceptable recoveries between $63 \%$ for STER to $95 \%$ for 270 OTA; however, in this study the mixture acetonitrile/methanol (50:50 v/v) improved 271 absolute recoveries for trichothecenes Type A $(108 \% \mathrm{DON}), \mathrm{AFs}\left(>66 \% \mathrm{AFB}_{2}\right)$ and 272 BEA $(>66 \%)$, as shown table 2. 
273

274 To evaluate the ability of the developed method for quantitative analysis of the selected

275 mycotoxins in beers, the analytical performance of the proposed method was studied

280

281

282

283

284

285

286

287

288

289

290

291

292

293

294

295

296

297 and validated in terms of linearity, limits of detection and quantification, precision and reproducibility of the technique. Validation experiments were performed with four types of beer brands available in Ireland: (i) stout beer, (ii) red ale beer, (iii) ale beer and (iv) pale lager beer. The analytical performance of the methodology for beer samples is summarized in table 2.

Limits of detection (LODs) for selected mycotoxins ranged from $3 \mathrm{ng} \mathrm{ml}^{-1}\left(\mathrm{AFB}_{1}\right.$ and $\mathrm{AFB}_{2}$ in ale and pale lager beers) to $50 \mathrm{ng} \mathrm{ml}^{-1}$ (NIV in stout beers). Lowest limits of quantification (LLOQs) ranged from $9 \mathrm{ng} \mathrm{ml}^{-1}\left(\mathrm{AFB}_{2}\right.$ in ale and pale lager beers) to 155 $\mathrm{ng} \mathrm{ml}^{-1}$ (NIV in stout beers). Although these limits did not differ significantly between the four matrices, in the case of stout and red ale beer seemed to be slightly higher. It could be assumed that this phenomenon is caused by the greater roasted of the malt or barley and the appearance of different new compounds that could interfere with the selected mycotoxins, increasing these limits.

Electrospray ionization is usually susceptible to matrix-related signal suppression, which is believed to result from the competition between the analyte ions and matrix components. Therefore the presence of co-extracted matrix components may affect analyte quantification. For this purpose, five calibration sets (standard in pure solvent, and matrix-matched standards of stout, red ale, ale and pale lager beer) at six concentration levels between LLOQ and 100 times LLOQ were prepared. As seen in table 2, matrix effects were calculated in percentages as the ratio of matrix-matched 
298 calibration slope and solvent calibration slope (Zachariasova et al. 2010). A value of

$299100 \%$ indicates that there is no absolute matrix effect. There exists signal enhancement

300 if the value is $>100 \%$ and signal suppression if the value is $<100 \%$. The matrix effects

301 did not differ significantly between the matrices selected. Severe suppression occurred 302 for aflatoxins, STER, trichothecenes type A (T-2 and HT-2) and trichothecenes type B 303 (3-ADON).

304

305 The best way to compensate the matrix effect is the use of isotopic internal standards.

306 Unfortunately, the labeled analogues or internal standard (IS) of these mycotoxins were 307 inaccessible to us owing to their cost (Romero- González et al. 2009). Thus, other 308 approaches were evaluated.

309

310 The first option was the addition of established internal standards used in mycotoxin 311 field as $\mathrm{AFM}_{1}, \mathrm{NEO}$ and 3-ADON-d $3 . \mathrm{AFM}_{1}$ and NEO are analogue molecules of AFs

312 and trichothecenes type A, respectively, since they are metabolites of these mycotoxins.

313 STER and AFs were calibrated with $\mathrm{AFM}_{1}$, since STER is supposed to be a precursor of 314 the AFs and $\mathrm{AFM}_{1}$ is a metabolite of $\mathrm{AFB}_{1} .3-\mathrm{ADON}-\mathrm{d} 3$ is the deutered analogue of 3315 ADON. With this calibration approach no significant improvement was achieved, 316 probably due to the fact that the optimal SPE-HPLC-LTQ-ORBITRAP conditions for 317 internal standards were different from those of the mycotoxins.

319 A second calibration alternative was tested according to Council decision 2002/657/EC

320 (European Community 2002) and document SANCO/10684 (2009): external matrix-

321 matched calibration. The use of matrix-matched standards compensated the suppression

322 signal effects achieving an accurate quantification as reported the literature (Bacaloni et 
323 al. 2005; Romero et al. 2009; Zachariasova et al. 2010). Matrix-matched calibration

324 curves were prepared in the four extract beers, showing good linearity between LLOQ 325 and 100 times LLOQ concentration levels (linear range), with a correlation coefficient $\geq$ $326 \quad 0.9861$.

328 The inter-day precision and intra-day repeatability of the method were also evaluated on 329 extracted beer samples at two different concentration levels (LLOQ and 100 times 330 LLOQ). The RSD values for intraday analyses were in the range 5-15\% and the RSD 331 for inter-day values were between 6 to $18 \%$, showing good reproducibility of the 332 technique. The recovery ranges in low and high spiked levels were $66-108 \%$ for the 333 intraday test and $65-103 \%$ for the inter-day test.

335 In the light of these results, the developed method gave acceptable recoveries and these 336 results were in good agreement with the performance criteria of Commission Regulation 337 (EC) $\mathrm{N}^{\mathrm{o}} 401$ of 23 February 2006 (European community 2006) laying down the 338 methods of sampling and analysis for the official control of the levels of mycotoxins in 339 foodstuffs.

341 Analysis of commercialized Irish beers.

342 In this study, the analyzed samples were divided into four main categories: stout, red 343 ale, ale and pale lager. Twenty-five samples were analysed: 6 stouts, 3 red ale, 6 ale and 34410 pale lager beers. The first step was to identify target compounds (selected 345 mycotoxins) in Irish beers. Red ale, ale and pale lager beers were analysed by LTQ 346 Orbitrap XL; in these alcoholic beverages selected mycotoxins were not present. 347 However, ZEN was identified and quantified (37.8 $\left.\mathrm{ng} \mathrm{ml}^{-1}\right)$ in one stout beer. Figure 1 
348 depicts a full scan of stout beer sample where ZEN was identified by retention time

349 (RT) (Figure 1A). After that, extracted ion chromatogram was checked for selected

350 compound and ZEN was observed at appropriate RT of 12.19 (Figure 1(b)). This

351 compound was unambiguously identified by accurate mass and pathway study. The

352 measured $\mathrm{m} / \mathrm{z}$ was 319.1547 and the calculated error (ppm) was 0.31 (Figure 1C). In

353 order to improve the identification of the mycotoxin, ion trap spectrum was acquired,

354 and characteristic pathway for ZEN was obtained. This mycotoxin (ZEN) lost one

355 molecule of water in the first fragmentation of ion trap $\left(\mathrm{MS}^{2}\right)$; this fragment has been

356 also reported by Frenich et al. (2009).

357

358 To our knowledge, this is the first time that ZEN is identified unambiguously by

359 accurate mass and fragmentation study in beer. Previous works that screened ZEN in

360 beer (Maragou et al. 2008; Romero et al. 2009) did not identify this mycotoxin in this

361 food commodity.

362

363 The second step was to screen "non-target" mycotoxins in Irish beer samples: enniatins

$364 \mathrm{~A}, \mathrm{~B}, \mathrm{~A}_{1}, \mathrm{~B}_{1}$ and fusaproliferin. The structures of these emergent mycotoxins were 365 studied (www.chemspider.com) and the exact mass was calculated (ChemDraw 11.0).

366 Several studies about emergent mycotoxins have reported two possibilities for

367 molecular ion formation; $[\mathrm{M}+\mathrm{H}]^{+}$and $\left[\mathrm{M}+\mathrm{NH}_{4}\right]^{+}$(Uhlig et al. 2004; Jestoi et al. 2009).

368 For this reason, exact mass for these ions were calculated and scanned on each sample

369 by Xcalibur 2.0 software. In this study these emergent mycotoxins were not identified.

370

371 Conclusion 
372 The SPE-LC-LTQ-Orbitrap-MS method reported in this paper was able to determine 18

373 mycotoxins in beer providing high sensitivity, high recoveries and achieving low LODs

374 and LLOQs. Moreover, this technology allowed a screening of 6 emergent mycotoxins,

375 non-target compounds which could be checked.

376

377 The developed method was applied for the analysis of selected mycotoxin in 25

378 different Irish beer samples. The results confirmed the presence of ZEN in one sample

379 of stout beer.

380

381 Even though it is true that the found concentration level was low, it is to be taken into

382 account that the risk of the exposition depends of the quantity of beer consumed for a

383 prolonged period of time; this makes necessary a mycotoxin control in beer production 384 chain.

385

386 To our knowledge, this work reports for the first time the application of LTQ-Orbitrap

387 technology to routine monitoring mycotoxins in commercial beers, demonstrating its

388 potential by accurate mass and pathway study.

389

390 Acknowledgements

391 This work was supported by the Spanish Ministry of Science and Innovation 392 (AGL2010-17024/ALI). CS thanks to Universitat de Valencia for the "short-term visit" 393 grant. 
396 Bacaloni A, Cavaliere C, Faberi A, Pastorini E, Samperi R, Lagana A. 2005. Automated

397 on-line solid-phase extraction-liquid chromatography-electrospray tandem mass

398 spectrometry method for the determination of ochratoxin A in wine and beer. $\mathbf{J}$ Agric

399 Food Chem 53:5518-5525.

400 Bennett KL, Funk M, Tschernutter M, Breitwieser FP, Planyavsky M, Mohien CU,

401 Müller A, Trajanoski Z, Colinge J, Superti-Furga G, Schmidt-Erfurth U. 2011.

402 Proteomic analysis of human cataract aqueous humour: Comparison of one-dimensional

403 gel LCMS with two-dimensional LCMS of unlabelled and iTRAQ®-labelled

404 specimens. J Proteomics 74: 151-166.

405 Document No. SANCO/10684. 2009. Method validation and quality control procedures

406 for pesticides residues analysis in food and feed.

407 Edison SE, Lin LA, Gamble BM, Wong J, Zhang K. 2011. Surface swabbing technique

408 for the rapid screening for pesticides using ambient pressure desorption ionization with

409 high-resolution mass spectrometry. Rapid Commun Mass Spectrom 25:127-139.

410 European Community. 2002. Council decision (2002)/657/EC. Off J Eur Comm L. $411 \quad 221: 8-36$.

412 European Community. 2006. Commission Regulation (EC) N 401/2006 of 23 February

4132006 laying down the methods of sampling and analysis for the official control of the

414 levels of mycotoxins in foodstuffs.

415 Frenich AG, Vidal JLM, Romero-González R, Aguilera-Luiz MdM. 2009. Simple and

416 high-throughput method for the multimycotoxin analysis in cereals and related foods by

417 ultra-high performance liquid chromatography/tandem mass spectrometry. Food Chem $418 \quad 117: 705-712$. 
419 Herebian D, Zühike S, Lamshöft M, Spiteller M. 2009. Multi-mycotoxin analysis in 420 complex biological matrices using LC-ESI/MS: Experimental study using triple stage 421 quadrupole and LTQ-Orbitrap. J Sep Sci 32:939-948.

422 Hogenboom AC, van Leerdam JA, de Voogt P. 2009. Accurate mass screening and 423 identification of emerging contaminants in environmental samples by liquid 424 chromatography-hybrid linear ion trap Orbitrap mass spectrometry. J Chromatogr A. $425 \quad 1216: 510-519$.

426 Jestoi M, Rokka M, Yli-Mattila T, Parikka P, Rizzo A, Peltonen K. 2004. Presence and 427 concentrations of the Fusarium-related mycotoxins beauvericin, enniatins and 428 moniliformin in Finnish grain samples. Food Addit Contam A 21:794-802.

429 Jestoi M. 2008. Emerging fusarium-mycotoxins fusaproliferin, beauvericin, enniatins, 430 and moniliformin - A review. Crit Rev Food Sci Nutr 48:21-49.

431 Jestoi M, Rokka M, Järvenpää E, Peltonen K. 2009. Determination of Fusarium 432 mycotoxins beauvericin and enniatins (A, A1, B, B1) in eggs of laying hens using liquid 433 chromatography-tandem mass spectrometry (LC-MS/MS). Food Chem 115:1120-1127. 434 Peters RJB, Stolker AAM, Mol JGJ, Lommen A, Lyris E, Angelis Y, Vonaparti A, 435 Stamou M, Georgakopoulos C, Nielen MWF. 2010. Screening in veterinary drug 436 analysis and sports doping control based on full-scan, accurate-mass spectrometry. 437 Trends Analyt Chem 29:1250-1268.

438 Makarov A, Scigelova, M. 2010. Coupling liquid chromatography to Orbitrap mass 439 spectrometry. J Chromatogr A. 1217:3938-3845.

440 Maragou NC, Rosenberg E, Thomaidis NS, Koupparis MA. 2008. Direct determination 441 of the estrogenic compounds 8-prenylnaringenin, zearalenone, $\alpha$ - and $\beta$-zearalenol in 442 beer by liquid chromatography-mass spectrometry. J Chromatogr A. 1202:47-57. 
443 Romero-González R, Vidal JLM, Aguilera-Luiz MM, Frenich AG. 2009. Application of

444 conventional solid-phase extraction for multimycotoxin analysis in beers by ultrahigh-

445 performance liquid chromatography-tandem mass spectrometry. J Agric Food Chem

$446 \quad 57: 9385-9392$.

447 Škrabáková Z, O'Halloran J, van Pelt FNAM, James KJ. 2010. Food contaminant 448 analysis at ultra-high mass resolution: Application of hybrid linear ion trap-orbitrap 449 mass spectrometry for the determination of the polyether toxins, azaspiracids, in 450 shellfish. Rapid Commun Mass Spectrom 24:2966-2974.

451 Thurman EM, Ferrer I, Zweigenbaum JA. 2006. High-resolution and accurate mass 452 analysis of xenobiotics in food. Anal Chem 78:6702-6708.

453 Uhlig S, Ivanova L. 2004. Determination of beauvericin and four other enniatins in 454 grain by liquid chromatography-mass spectrometry. J Chromatogr A. 1050:173-178.

455 Vaclavik L, Zachariasova M, Hrbek V, Hajslova J. 2010. Analysis of multiple 456 mycotoxins in cereals under ambient conditions using direct analysis in real time 457 (DART) ionization coupled to high resolution mass spectrometry. Talanta 82:19504581957.

459 Van der Heeft E, Block YJC, Beumer B, Nijrolder AWJM, Stolker AAM, Nielen MWF. 460 2009. Full-scan accurate mass selectivity of ultra-performance liquid chromatography 461 combined with time-of-flight and orbitrap mass spectrometry in hormone and veterinary 462 drug residue analysis. J. Am. Soc. Mass Spectrom. 20:451-463.

463 Zachariasova M, Cajka T, Godula M, Malachova A, Veprikova Z, Hajslova J. 2010. 464 Analysis of multiple mycotoxins in beer employing (ultra)-high-resolution mass 465 spectrometry. Rapid Commun Mass Spectrom 24:3357-3367. 


\section{FIGURE CAPTION:}

467 Figure 1: Chromatogram of positive stout beer sample: A) Full scan MS 100-900 m/z.

468 B) extracted ions $m / z$ 319,15. C) accurate mass ZEN and D) confirmation by ion trap 469 (MS2).

470

471

472 
Table 1. Ion formation, elemental formula, theoretical and measured $\mathrm{m} / \mathrm{z}$ and error mass (ppm) for selected mycotoxins. Data-dependingacquisition parameters for ion trap acquisition.

\begin{tabular}{|c|c|c|c|c|c|c|c|c|}
\hline \multirow{2}{*}{ Compound } & \multicolumn{5}{|c|}{ Full Scan FTMS } & \multicolumn{3}{|c|}{ Data-depending-acquisition (DDA) } \\
\hline & Ion formation & Elemental formula & Theoretical $\mathrm{m} / \mathrm{z}$ & Measured $\mathrm{m} / \mathrm{z}$ & Error (ppm) & Retention time & Parent mass & $\mathrm{CE}$ \\
\hline NIV & {$[\mathrm{M}+\mathrm{H}]^{+}$} & $\mathrm{C}_{15} \mathrm{H}_{21} \mathrm{O}_{7}$ & 313.1287 & 313.1289 & 0.63 & 4.99 & 313.13 & 18 \\
\hline DON & {$[\mathrm{M}+\mathrm{H}]^{+}$} & $\mathrm{C}_{15} \mathrm{H}_{21} \mathrm{O}_{6}$ & 297.1338 & 297.1337 & -0.33 & 6.35 & 297.13 & 20 \\
\hline 3-ADON & {$[\mathrm{M}+\mathrm{H}]^{+}$} & $\mathrm{C}_{17} \mathrm{H}_{23} \mathrm{O}_{7}$ & 339.1445 & 339.1444 & -0.29 & 8.50 & 339.14 & 18 \\
\hline DAS & {$\left[\mathrm{M}+\mathrm{NH}_{4}\right]^{+}$} & $\mathrm{C}_{19} \mathrm{H}_{30} \mathrm{O}_{7} \mathrm{~N}$ & 384.2023 & 384.2021 & -0.52 & 8.48 & 383.20 & 19 \\
\hline HT-2 & {$\left[\mathrm{M}+\mathrm{NH}_{4}\right]^{+}$} & $\mathrm{C}_{22} \mathrm{H}_{36} \mathrm{O}_{8} \mathrm{~N}$ & 442.2441 & 442.2445 & 0.90 & 10.81 & 442.24 & 18 \\
\hline $\mathrm{T}-2$ & {$\left[\mathrm{M}+\mathrm{NH}_{4}\right]^{+}$} & $\mathrm{C}_{24} \mathrm{H}_{38} \mathrm{O}_{9} \mathrm{~N}$ & 484.2547 & 484.2545 & -0.55 & 11.34 & 484.25 & 18 \\
\hline $\mathrm{FB}_{1}$ & {$[\mathrm{M}+\mathrm{H}]^{+}$} & $\mathrm{C}_{34} \mathrm{H}_{60} \mathrm{O}_{15} \mathrm{~N}$ & 722.3963 & 722.3962 & -0.13 & 10.45 & 722.39 & 54 \\
\hline $\mathrm{FB}_{2}$ & {$[\mathrm{M}+\mathrm{H}]^{+}$} & $\mathrm{C}_{34} \mathrm{H}_{60} \mathrm{O}_{14} \mathrm{~N}$ & 706.4014 & 706.4017 & 0.42 & 11.62 & 706.40 & 25 \\
\hline $\mathrm{FB}_{3}$ & {$[\mathrm{M}+\mathrm{H}]^{+}$} & $\mathrm{C}_{34} \mathrm{H}_{60} \mathrm{O}_{14} \mathrm{~N}$ & 706.4014 & 706.4016 & 0.28 & 11.06 & 706.40 & 25 \\
\hline ZEN & {$[\mathrm{M}+\mathrm{H}]^{+}$} & $\mathrm{C}_{18} \mathrm{H}_{23} \mathrm{O}_{5}$ & 319.1546 & 319.1547 & 0.31 & 12.19 & 319.15 & 20 \\
\hline $\mathrm{ZOL}$ & {$[\mathrm{M}+\mathrm{H}]^{+}$} & $\mathrm{C}_{18} \mathrm{H}_{25} \mathrm{O}_{5}$ & 321.1702 & 321.1705 & 0.93 & 11.95 & 321.17 & 20 \\
\hline $\mathrm{AFB}_{1}$ & {$[\mathrm{M}+\mathrm{H}]^{+}$} & $\mathrm{C}_{17} \mathrm{H}_{13} \mathrm{O}_{6}$ & 313.0711 & 313.0712 & 0.31 & 9.78 & 313.07 & 35 \\
\hline $\mathrm{AFB}_{2}$ & {$[\mathrm{M}+\mathrm{H}]^{+}$} & $\mathrm{C}_{17} \mathrm{H}_{15} \mathrm{O}_{6}$ & 315.0869 & 315.0868 & -0.31 & 9.63 & 315.08 & 35 \\
\hline $\mathrm{AFG}_{1}$ & {$[\mathrm{M}+\mathrm{H}]^{+}$} & $\mathrm{C}_{17} \mathrm{H}_{13} \mathrm{O}_{7}$ & 329.0661 & 329.0662 & 0.30 & 9.30 & 329.06 & 29 \\
\hline $\mathrm{AFG}_{2}$ & {$[\mathrm{M}+\mathrm{H}]^{+}$} & $\mathrm{C}_{17} \mathrm{H}_{15} \mathrm{O}_{7}$ & 331.0818 & 331.0816 & -0.60 & 9.10 & 331.08 & 32 \\
\hline STER & {$[\mathrm{M}+\mathrm{H}]^{+}$} & $\mathrm{C}_{18} \mathrm{H}_{13} \mathrm{O}_{6}$ & 325.0712 & 325.0711 & -0.30 & 12.43 & 325.07 & 35 \\
\hline OTA & {$[\mathrm{M}+\mathrm{H}]^{+}$} & $\mathrm{C}_{20} \mathrm{H}_{19} \mathrm{O}_{6} \mathrm{NCl}$ & 404.0901 & 404.0903 & 0.43 & 12.23 & 404.09 & 17 \\
\hline BEA & {$\left[\mathrm{M}+\mathrm{NH}_{4}\right]^{+}$} & $\mathrm{C}_{45} \mathrm{H}_{61} \mathrm{O}_{9} \mathrm{~N}_{4}$ & 801.4439 & 801.4437 & -0.24 & 14.01 & 801.44 & 50 \\
\hline
\end{tabular}


Table 2. Matrix effect, lowest limits of quantification (LLOQs), recovery values (RSD \%) given in brackets calculated at two concentration levels $\left(\mathrm{ng} \mathrm{ml}^{-1}\right)$, mean level for all four matrices is presented. Results show values for stout/red ale/ale/pale lager beers.

\begin{tabular}{|c|c|c|c|c|c|c|c|}
\hline & & & & \multicolumn{2}{|c|}{ Intr-day precision } & \multicolumn{2}{|c|}{ Inter-day precision } \\
\hline Compound & Matrix effect $(\%)$ & $\begin{array}{c}\mathrm{LOD} \\
\left(\mathrm{ng} \mathrm{ml}^{-1}\right)\end{array}$ & $\begin{array}{c}\text { LLOQ } \\
\left(\mathrm{ng} \mathrm{ml}^{-1}\right)\end{array}$ & $\begin{array}{c}\text { Low level } \\
\text { (LLOQ) }\end{array}$ & $\begin{array}{l}\text { High level } \\
\text { (100xLLOQ) }\end{array}$ & $\begin{array}{c}\text { Low level } \\
\text { (LLOQ) }\end{array}$ & $\begin{array}{c}\text { High level } \\
\text { (100xLLOQ) }\end{array}$ \\
\hline NIV & 90/98/94/98 & $50 / 45 / 44 / 40$ & $155 / 135 / 131 / 120$ & 77/80/84/91 (10) & 78/82/84/89 (10) & 73/81/80/90 (10) & 70/81/84/89 (11) \\
\hline DON & $83 / 105 / 85 / 94$ & $20 / 18 / 16 / 16$ & $60 / 54 / 48 / 48$ & $83 / 84 / 87 / 108(11)$ & 80/81/86/103 (9) & 78/83/81/97 (11) & $81 / 81 / 83 / 100(9)$ \\
\hline 3-ADON & $43 / 55 / 48 / 59$ & $30 / 27 / 25 / 25$ & $90 / 80 / 75 / 75$ & $83 / 84 / 87 / 108(11)$ & $82 / 86 / 85 / 102(8)$ & $80 / 84 / 85 / 101(12)$ & $82 / 85 / 85 / 102(9)$ \\
\hline DAS & $107 / 101 / 102 / 102$ & $13 / 13 / 12 / 10$ & $40 / 40 / 35 / 30$ & 93/96/95/103 (9) & 95/96/98/101 (5) & $92 / 95 / 95 / 102(10)$ & 98/98/98/103 (9) \\
\hline HT-2 & $59 / 63 / 53 / 65$ & 7/9/7/7 & $20 / 27 / 20 / 20$ & 71/75/77/79 (9) & 73/78/79/79 (6) & 72/78/79/81 (10) & 76/80/81/85 (11) \\
\hline $\mathrm{T}-2$ & $63 / 69 / 63 / 68$ & $5 / 5 / 4 / 4$ & $15 / 15 / 12 / 12$ & 68/76/74/92 (10) & 71/79/78/99 (8) & 68/69/71/83 (10) & $68 / 72 / 72 / 89(11)$ \\
\hline $\mathrm{FB}_{1}$ & $115 / 111 / 99 / 112$ & $35 / 32 / 30 / 32$ & $105 / 95 / 90 / 95$ & $80 / 85 / 87 / 92(11)$ & 77/79/80/89 (9) & $81 / 81 / 82 / 89(12)$ & $81 / 80 / 80 / 89(10)$ \\
\hline $\mathrm{FB}_{2}$ & $100 / 125 / 111 / 109$ & $32 / 32 / 30 / 30$ & 95/95/90/90 & $85 / 89 / 87 / 88(10)$ & $80 / 82 / 83 / 85(8)$ & $86 / 88 / 87 / 88(15)$ & $80 / 81 / 80 / 86(14)$ \\
\hline $\mathrm{FB}_{3}$ & $116 / 112 / 119 / 123$ & $32 / 32 / 32 / 32$ & 95/95/95/95 & $82 / 85 / 79 / 91(12)$ & 78/80/75/81 (11) & $83 / 83 / 81 / 88(15)$ & 77/79/76/78 (11) \\
\hline ZEN & $87 / 79 / 82 / 83$ & $12 / 10 / 12 / 10$ & $36 / 30 / 36 / 30$ & 75/77/85/88 (10) & $72 / 74 / 85 / 83(8)$ & 77/77/81/85 (11) & $71 / 70 / 81 / 82(7)$ \\
\hline $\mathrm{ZOL}$ & $90 / 88 / 89 / 93$ & $10 / 10 / 8 / 8$ & $30 / 30 / 24 / 24$ & 75/81/85/91 (8) & $72 / 78 / 78 / 89(5)$ & 73/83/82/90 (9) & $73 / 75 / 76 / 88(5)$ \\
\hline $\mathrm{AFB}_{1}$ & $56 / 60 / 59 / 61$ & $5 / 4 / 3 / 3$ & $15 / 12 / 9 / 9$ & 77/79/73/78 (5) & 79/79/78/81 (5) & 78/79/76/80 (7) & 77/79/77/78 (9) \\
\hline $\mathrm{AFB}_{2}$ & $55 / 56 / 65 / 64$ & $4 / 3 / 3 / 3$ & $12 / 10 / 9 / 9$ & 66/76/71/81 (8) & $72 / 79 / 75 / 83(6)$ & $66 / 71 / 72 / 82(10)$ & 71/78/72/80 (9) \\
\hline $\mathrm{AFG}_{1}$ & $51 / 57 / 67 / 65$ & $5 / 4 / 4 / 4$ & $15 / 12 / 12 / 12$ & 70/73/71/79 (7) & 76/75/77/79 (7) & 70/70/70/79 (7) & $72 / 71 / 72 / 81(8)$ \\
\hline $\mathrm{AFG}_{2}$ & $56 / 56 / 69 / 61$ & $4 / 5 / 4 / 4$ & $12 / 15 / 12 / 12$ & 68/69/72/77 (9) & 70/76/76/79 (6) & $68 / 68 / 70 / 75(9)$ & 71/75/74/78 (9) \\
\hline STER & $66 / 67 / 69 / 71$ & $7 / 6 / 6 / 5$ & $21 / 18 / 18 / 15$ & 69/79/72/81 (6) & 72/80/76/84 (7) & $65 / 73 / 76 / 80(6)$ & 71/77/75/87 (9) \\
\hline OTA & 77/71/75/79 & $5 / 4 / 4 / 4$ & $15 / 12 / 12 / 12$ & 70/77/75/94 (10) & 78/82/85/97 (9) & 71/72/78/89 (12) & $79 / 80 / 81 / 90(8)$ \\
\hline BEA & $55 / 66 / 59 / 64$ & $4 / 5 / 5 / 4$ & $12 / 15 / 15 / 12$ & 69/75/91/99 (15) & 71/73/88/93 (13) & 66/77/88/93 (18) & 70/73/90/95 (16) \\
\hline
\end{tabular}

${ }^{\mathrm{a}}$ Number of replicates:5

${ }^{\mathrm{b}}$ Different days: 5 

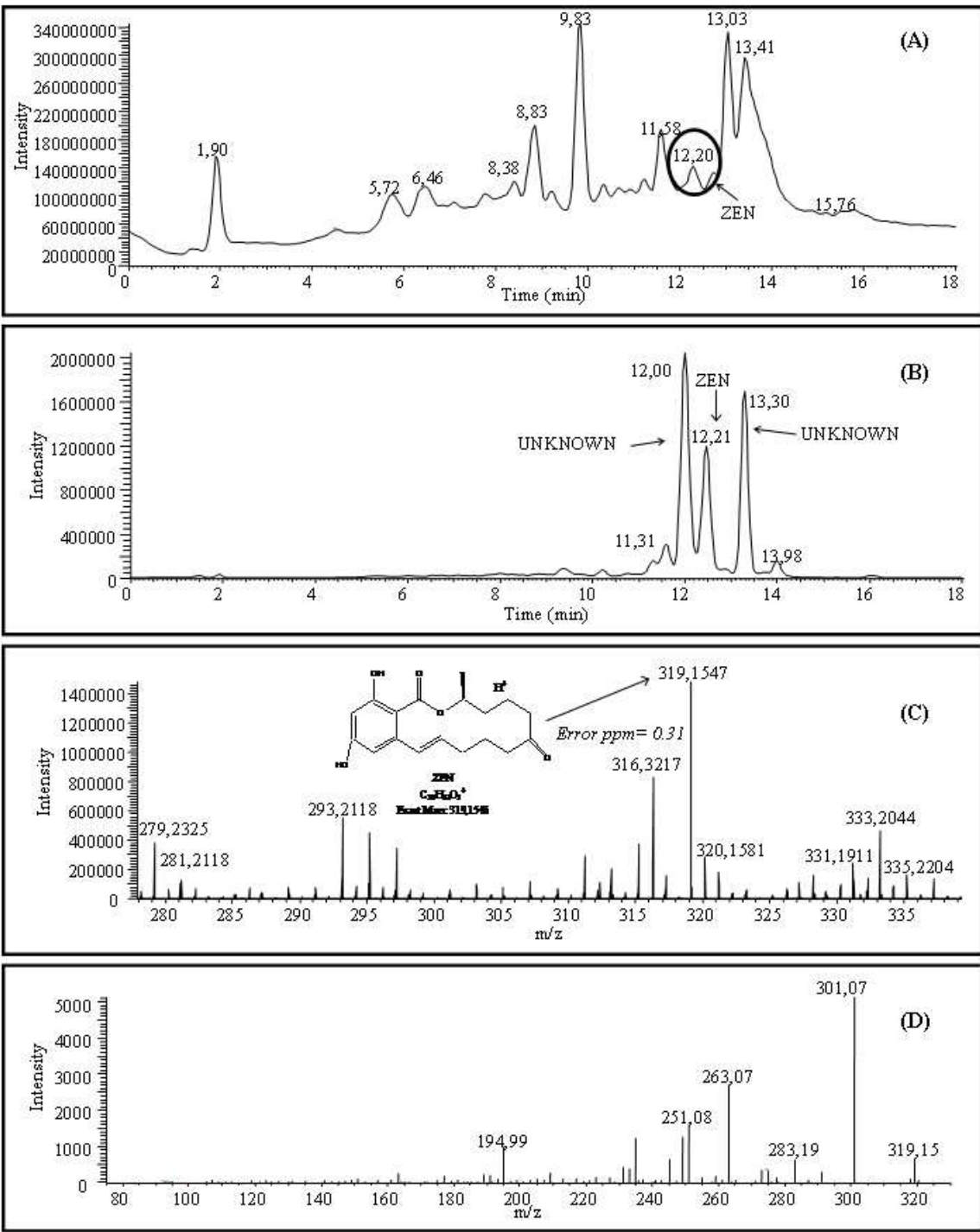

Figure 1. Chromatogram of positive stout beer sample: A) Full scan MS 100-900 m/z. B) extracted ions $m / z 319,15$. C) accurate mass $Z E N$ and $D)$ confirmation by ion trap $\left(M^{2}\right)$.

$190 \times 254 \mathrm{~mm}(96 \times 96$ DPI)

http://mc.manuscriptcentral.com/tfac Email: fac@tandf.co.uk 